\title{
PENÉLOPE E HIPERMESTRA, EXEMPLA UXORIS: DISPOSITIO Y EJEMPLARIDAD DE LAS HEROIDAS 1 Y 14, DE OVIDIO
}

Cecilia Marcela Ugartemendía ${ }^{1}$

RESUMEN: El objetivo de este artículo es justificar la dispositio de las Ep. 1 (de Penélope a Ulises) y 14 (de Hipermestra a Linceo) en el corpus transmitido en la actualidad. El propósito es demostrar que las epístolas 1 y 14, gracias a las características paradigmáticas de las mujeres que las escriben, son llaves de apertura y clausura de una secuencia de exempla mitológicos sobre el buen comportamiento de la mujer en una relación amorosa. Además, discutiremos cómo Cánace, en la Ep. 11, se apropia intratextualmente de las virtudes de estas dos mujeres ejemplares para argumentar en su propia defensa. Al admitir sus errores, enfatiza la importancia de virtudes como la castitas, la pudicitia y la pietas, mejor representadas en el corpus por Penélope e Hipermestra.

PALABRAS ClaVE: Heroidas; intratextualidad; Penélope; Hipermestra; Cánace.

ABSTRACT: This article discusses the dispositio of Ep. 1 (from Penelope to Ulysses) and Ep. 14 (from Hypermnestra to Lynceus) in the transmitted corpus. The purpose is to demonstrate that the epistles 1 and 14, due to the paradigmatic features of the women who write them, are key elements in the opening and closure in the sequence of mythological exempla on women's behaviour in a love relationship. Plus, we will discuss how Canace, Ep. 11, assumes intratextually - virtues of the two exemplary women for herself to argue her own defense. By admitting her mistakes, she emphasizes the importance of virtues such as castitas, pudicitia and pietas, best represented in the corpus by Penelope and Hypermnestra.

KEYWORDS: Heroides; intratextuality; Penelope; Hypermnestra; Canace.

\section{Introducción}

Heroidas es una obra de la fase inicial de la carrera poética de Ovidio. La mayoría de los críticos la posiciona después de la primera publicación de los Amores. Sin embargo, Knox (1996, p. 6) nota que esta colección de epístolas elegíacas no solo antecede la segunda edición de dicha obra (2 a.C.), sino que, posiblemente, incluso la primera (16 a.C.). Por esto, el crítico deduce que la

\footnotetext{
${ }^{1}$ La autora es Magíster en Letras Clásicas por la Universidad de São Paulo y doctoranda en el programa de Pos-graduación en Letras Clásicas en la misma institución. Es becaria de doctorado de la FAPESP, en convenio con la CAPES (Proceso 2017/01934-8). Agradecemos a estas instituciones por el apoyo financiero. E-mail: cecilia.7u@usp.br
} 
fecha de publicación más probable (al menos de la mayor parte de los primeros quince poemas) oscila entre el 25 y 16 a.C.

Este corpus se compone de 21 epístolas, divididas en epístolas simples y dobles. Se conocen como simples las primeras quince, cuyos remitentes son mujeres pertenecientes a la tradición poética y mítica que escriben a sus amados ausentes, procurando convencerlos de que retornen junto a ellas. ${ }^{2}$ Las últimas seis, conocidas como dobles, son un intercambio epistolar entre tres parejas de la tradición mítica. 3

La transmisión textual de las Heroidas es problemática. Esto se debe a la cantidad de manuscritos (en los más de doscientos conocidos raramente se encuentran dos versiones iguales), que ofrecen múltiples lecturas, variaciones y lectiones. Escribas, copistas y enmendadores interpretaron y corrigieron sus versos, haciendo de esta colección una obra obscura para la edición textual.

El mayor problema se encuentra en la Ep. 15, de Safo a Faón, y en las epístolas dobles. En los mejores manuscritos de estas últimas se omiten importantes pasajes. De hecho, la crítica concuerda en que, dadas las anomalías en la elocución y métrica y el cambio en el tratamiento de los tópicos, las epístolas dobles pertenecerían o bien a la misma época que los poemas del exilio ovidiano, o a un período posterior al clásico, o bien a una obra diferente, inclusive no ovidiana. 4 Por otro lado, la Ep. 15, de Safo a Faón, presenta problemas de transmisión por ser independiente del resto de las Heroidas. Apenas sobrevive en un manuscrito medieval - además de en algunos florilegios - en el que está colocada en primer lugar, precediendo a las demás. Ovidio en Amores (2.18.26) cuenta que está componiendo las epístolas de una serie de heroínas, entre las que, en último lugar, se menciona a Safo. Daniel Heinsius, en su edición del siglo XVII, guiándose por este poema, coloca la Ep. 15 a continuación de las otras. A él se debe la posición de esta epístola en el último lugar de la colección. 5

\footnotetext{
2 Penélope a Ulises, Ep. 1; Filis a Demofonte, Ep. 2; Briseida a Aquiles, Ep. 3; Fedra a Hipólito, Ep. 4; Enone a Paris, Ep. 5; Hipsípila a Jasón, Ep. 6; Dido a Eneas, Ep. 7; Hermíone a Orestes, Ep. 8; Deyanira a Hércules, Ep. 9; Ariadna a Teseo, Ep. 10; Cánace a Macareo, Ep. 11; Medea a Jasón, Ep. 12; Laodamia a Protesilao, Ep. 13; Hipermestra a Linceo, Ep. 14 y Safo a Fáon, Ep. 15. 3 Paris a Helena, Ep. 16 - Helena a Paris, Ep. 17; Leandro a Hero, Ep. 18 - Hero a Leandro, Ep. 19 y Aconcio a Cídipe, Ep. 20 - Cídipe a Aconcio, Ep. 21.

4 Sobre esto, véase Jacobson (1974, p. ix); Kenney (1996, p. 21-25); Farrel (1998); Michalopoulos (2006, p. 1ss). Sobre la posible datación y autenticidad, véase más en Knox (2002, p. 117-139).

5 Los problemas de la transmisión de las Heroidas fueron exhaustivamente estudiados por Dörrie (1960) en un libro dedicado exclusivamente a la transmisión de esta obra y en su propia
} 
No obstante, dejando de lado la epístola de Safo y las particularidades hasta ahora mencionadas, hay consenso en la disposición de los primeros catorce poemas del corpus, compatible con una coherencia interna que permite, incluso, intrarrelacionarlas. Así lo demuestran los trabajos de Stroh (1991, p. 201-244) y Holzberg (1997, p. 84ss.), quienes proponen diferentes formas de agrupar las epístolas según sus relaciones. Conforme estos autores, las quince cartas se pueden dividir en tres grupos. Para Holzberg, cada grupo tiene un tema como hilo conductor: el primero, la esperanza; el segundo, la resignación; el tercero, la desesperación.

A partir de esta tripartición en grupos temáticos, Stroh, por su parte, propone correspondencias que surgen a partir de la posición de las epístolas en el corpus. Según esta propuesta, el orden paralelo de las epístolas de los primeros dos grupos deja en evidencia la correspondencia temática. De este modo, Penélope (Ep. 1: primera epístola del primer grupo) e Hipsípila (Ep. 6: primera epístola del segundo grupo) son ejemplos de fieles esposas dejadas atrás por sus maridos; Filis (Ep. 2: segunda del primer grupo) y Dido (Ep. 7: segunda del segundo grupo), abandonadas por el extranjero a quien hospedaron, eligen la muerte para el fin de sus tristezas, etc.

A estos dos grupos sigue un tercero que - Stroh arriesga - puede haber sido publicado posteriormente y presenta un plan de ordenamiento que tiene un efecto de contraste con los primeros. A las fieles y convencionales esposas Penélope e Hipsípila se contrapone la incestuosa Cánace (Ep. 11: primera del tercer grupo); Medea (Ep. 12: segunda del segundo grupo), en vez de suicidarse preservando su honra, como las otras dos extranjeras a quienes se contrapone perversamente (Filis y Dido), prefiere buscar venganza a través del filicidio y así sucesivamente.

A continuación, buscamos sumar un argumento a la defensa de la dispositio con la que el corpus es transmitido en la actualidad. Para esto, nos centraremos en la coherencia de la ubicación de la Ep. 1, de Penélope a Ulises, y la Ep. 14, de Hipermestra a Linceo. Mediante este análisis, buscamos demostrar que estas dos epístolas funcionan como apertura y clausura, respectivamente, de la secuencia conformada por las primeras catorce epístolas, que funcionan como

edición de las Heroidas, Dörrie (1971), que goza hasta hoy de gran autoridad frente a la crítica y presenta la singularidad de reunir las 21 epístolas. Ver también Luck (1969), quien dedica un libro al estudio de la transmisión de Ovidio y propone stemmata de cada una de las obras. 
exempla mitológicos sobre el comportamiento esperable en una relación amorosa. Las dos representan modelos paradigmáticos, resaltando su pudicitia, castitas y pietas. Ese modelo se ve reflejado intratextualmente en la epístola de Cánace (Ep. 11). Ella también recurre a la pietas para pedir por su hijo a cambio de su vida, pero de forma diferente a Hipermestra y Penélope. Su comportamiento, aunque no sea ideal, se ve recompensado por la decisión de acatar la orden paterna, probando su pietas y resaltando al menos una de las virtudes que la asemeja a los paradigmas. Nuestro interés en la epístola de Cánace y su relación con las otras dos surge como posible alternativa a la interpretación propuesta por Fulkerson (2005, p. 67-86), que desarrollaremos en la sección correspondiente.

\section{Coherencia $y$ dispositio}

Las mujeres que forman parte de las Heroidas tienen carácter paradigmático. Sus historias, en efecto, son utilizadas a modo de exempla tanto por Ovidio como por otros autores de la tradición poética anterior a él. En el libro 3 de la Ars amatoria (3.339-346), el praeceptor recomienda a sus discípulas conocer su colección de epístolas como parte de su aprendizaje. Inclusive, él mismo en diferentes ocasiones se refiere a las mujeres que 'escriben' en las Heroidas como exempla del fracaso en la ars amandi. Por esta razón, el corpus de epístolas puede entenderse como una serie de ejemplos destinados al lector/discípulo y funciona como un complemento para el propósito didáctico del poema. Por causa de la falta de este tipo de ars, las heroínas fracasan al intentar convencer a sus amantes de volver junto a ellas. Es por eso que el lector recibe las epístolas como un gran exemplum de aquello que no se debe hacer en una relación amorosa y como justificativa de la necesidad de un praeceptor. ${ }^{6}$

La serie de exempla de comportamientos se encuentra enmarcada por las epístolas de Penélope (Ep. 1) e Hipermestra (Ep. 14), mujeres óptimas en su rol de esposas y entre cuyas cartas se reconocen numerosos puntos de contacto. Por un lado, la ejemplaridad de Penélope, presente ya en sus características personales, se refuerza gracias a su posición de privilegio en esta obra, al

\footnotetext{
${ }^{6}$ Véase Ugartemendía (2017), en donde esta hipótesis se desarrolla por extenso.
} 
inaugurar el ignotum opus7 ovidiano - la epístola elegíaca. Su carta tiene una función programática, delineando los temas que serán desarrollados, en distinta medida, en las epístolas subsecuentes. Su persona se identifica fácilmente con la épica, género mayor que cualquier otro, ya que ella entra en la poesía por medio de la épica homérica y, desde entonces, representa el ideal de mujer púdica ${ }^{8}$ y fiel. Penélope, pues, la más noble y paradigmática entre las mujeres, es la primera tanto en el corpus de las Heroidas como en la tradición poética. 9

Conscientes del carácter intertextual de sus personae, las heroínas, en sus epístolas, dialogan con la tradición poética de la que forman parte. Debido a esto, Penélope, autoconsciente de su ejemplaridad, apela a ese carácter paradigmático como argumento para convencer al héroe Ulises a regresar con ella. Para tanto, en su carta da por sentadas las razones por la cuales merece salir airosa en su cometido de convencerlo a retornar lo más rápido posible.

La ejemplaridad de Penélope será resaltada por el poeta también en el Ars amatoria, donde utilizará la figura de la pia Penelope como primer exemplum de mujer virtuosa (Ov. Ars 3.15-16). Al establecer un puente autotextual entre las Heroidas y el Ars amatoria, la epístola de Penélope complementa el exemplum presentado por el praeceptor y justifica la importancia de su posición como umbral del corpus epistolar.

Hipermestra, por su parte, se caracteriza por su cumplimiento de la pietas debida al marido. Ella organiza la carta como una defensa ante la acusación de su padre Dánao, propia del ámbito forense. Él les había ordenado tanto a ella como a sus hermanas que mataran a sus maridos en la misma noche de bodas, orden que solo Hipermestra no acató. De ahí que ella dirija la carta a Linceo, su flamante marido, pidiéndole ayuda, puesto que fue condenada a muerte por su propio padre a causa de su desobediencia. Aun así, cabe notar

\footnotetext{
7 Así es como el poeta se refiere a las Heroidas en Ov. Ars 3.345-346: Vel tibi composita cantetur Epistula voce: / Ignotum hoc aliis ille novavit opus ["o canta, con voz apropiada, una Epístola: él inventó este género desconocido por otros”]. Todas las traducciones del latín son propias.

8 Esta palabra, en todas sus apariciones en el texto, se utilizó teniendo en cuento su sentido etimológico.

9 Las características de Penélope son compatibles con las listadas por Semónides de Amorgos en el final de la sátira contra las mujeres (fr. 7 West). En este poema de carácter fuertemente misógino, el poeta presenta un catálogo de diez tipos de mujeres, dos basadas en elementos de la naturaleza y ocho en un tipo de animal. El último es el único tipo descrito de forma positiva. Esta es la mujer proveniente de la abeja, cuya naturaleza ejemplar remite fácilmente al paradigma representado por Penélope en la tradición poética.
} 
que el contenido de su epístola es llamativo, pues parece estar dirigida no solo a Linceo, sino también, de forma latente, a Dánao. Fulkerson (2005, p. 124), al notar esto, afirma que la epístola tiene no solo dos destinatarios explícitos, sino también dos objetivos: persuadir al marido para que la rescate y convencer a su padre para que no la castigue, delineando un discurso propio del ámbito forense. ${ }^{10}$ En la epístola, Hipermestra apela a los elementos emotivos del discurso, buscando demostrar su carácter probo y teniendo siempre, como base de su defensa, la pietas y la contradicción en su acusación. En efecto, lo más peculiar de su caso es el hecho de estar acusada por ser pia. Esto le servirá como argumento a favor en toda su epístola.

$\mathrm{Al}$ igual que Penélope, Hipermestra también sirve como exemplum a los elegíacos (véase, por ejemplo, Propercio 4.7.63: sine fraude marita), pero también a Horacio, quien en la Oda 3.11.33-36 dedica versos a su figura y la califica como virgo nobilis. La epístola de esta heroína ejemplar cierra la serie de las primeras catorce Heroidas, todas ellas escritas, como ya dijimos, por mujeres pertenecientes a la tradición mítica.

Penélope e Hipermestra, exempla uxoris

La epístola de Hipermestra se inicia con un dístico de cuya autenticidad sospechan muchos editores, a pesar de encontrarse atestiguado en todos los manuscritos: ${ }^{11}$

Mittit Hypermestra de tot modo fratribus uni;

Cetera nuptarum crimine turba iacet (Ovidio, Epistulae heroidum, 14.1-2).12

[“Hipermestra le envía al único de, hasta poco atrás, tantos hermanos; el resto del grupo yace por el crimen de las esposas.”]

De tot modo fratribus uni señala la peculiaridad del destinatario y funciona como primer argumento persuasivo de Hipermestra, recalcando el

\footnotetext{
${ }^{10}$ Este discurso fue analizado en Ugartemendía (2014).

${ }^{11}$ Las razones para no aceptar este dístico tienen que ver con su sentido, cuyo traslado a otro lugar de la epístola o su anulación tendría como resultado que la carta se iniciara de la misma forma que Ep. 8. Según Reeson (2001, p. 212-213), es posible que el primer dístico se encuentre perdido y este sea en verdad el segundo, dado que Hipermestra se dirige a Linceo como uno de los hijos de Egipto y no como su marido. No obstante, no todas las epístolas se inician resaltando la relación formal que el amado tiene con la mujer. Sea como fuere, no hay consenso sobre el carácter espurio del dístico.

${ }_{12}$ Todas las citas del texto latino de las Heroidas pertenecen a la edición de Ehwald (1907).
} 
hecho de la singularidad de su supervivencia. En el verso 3, Hipermestra declara estar encerrada en la domus (clausa domo teneor grauibusque coercita uinclis [“soy mantenida encerrada en la prisión y atada con pesadas cadenas”]). Toda la acción de Hipermestra ocurre en el espacio interno de la domus. Con todo, esa reclusión es llevada a un nivel hiperbólico, ya que, aquí, domus, como vertido en la traducción, puede tener la acepción de "prisión". ${ }^{13} \mathrm{El}$ primer adjetivo con el que ella se califica a sí misma es clausa (v. 3), condición de la que se enorgullece al declarar esse ream praestat [v. 7, "es preferible ser rea"]. Esta será la principal diferencia con Cánace, otra puella también clausa en su domus por el propio padre.

De la misma manera, en el comienzo del corpus, Penélope está retenida en su propia domus, esperando el retorno del marido que debe ir a rescatarla de veinte años de espera y de la presión ejercida por su padre, Icario, quien insiste en que contraiga nuevas nupcias. La reclusión de Penélope en los muros de la propia casa va de la mano de su identidad de mujer leal:

que Penélope sea descrita ya en su habitación, ya en el comedor público con los pretendientes es una elección del poeta; sin embargo, Penélope debe permanecer entre los corredores de su casa o perder su tradicional identidad de esposa leal [...]. Penélope no se mueve fuera de su casa; de hecho, su primera identificación espacial es sola en su cama desierta: non ego deserto iacuissem frigida lecto [Ep. 1.7] [...]. Remueve a Penélope de su espacio y ella, según su punto de vista, no será más su esposa. Penélopeen-su-cama es quien ella es (Bolton, 2009, p. 274-275).14

La devoción por el marido implica la restricción del espacio permitido a la mujer. Por tanto, la reclusión de la mujer, ya desde la primera epístola, indica un modo de virtud femenina.

Hipermestra defiende su decisión de no haber asesinado a Linceo argumentando el cumplimiento de la pietas debida al marido. Sobre este asunto, Jacobson (1974, p. 125) observa que pietas, tanto en su forma adjetiva

\footnotetext{
13 Como también en Prop. 2.20.10, 12.

14 "Whether Penelope is depicted in her bedroom or in the public hall with the suitors is the poet's choice, yet Penelope must remain within the halls of the house or lose her traditional identity as a loyal wife [...]. Penelope does not move outside of her house; indeed, her first spatial identification of herself is alone in her deserted bed: non ego deserto iacuissem frigida lecto [...]. Remove Penelope from her space and she, in her view, will no longer be his wife. Penelope-in-her-bed is who Penelope is”. Todas las traducciones del inglés son propias.
} 
como substantiva, aparece siete veces en los 132 versos de la epístola de Hipermestra, mientras que no más de tres en cualquier otra. Además, apenas dos de las otras mujeres en el corpus mencionan la propia pietas. La primera de estas otras dos instancias de aparición está en la epístola de Penélope (Ep. 1. 84), quien, bajo presión de su padre para casarse nuevamente, declara su devoción al marido: Penelope coniunx semper Ulixis ero [“yo, Penélope, siempre seré la esposa de Ulises”]). ${ }^{15}$ Este verso se encuentra casi al final de la epístola, en medio de la sospecha de que Ulises todavía puede estar lejos, pero de forma deliberada. Por eso, la esposa precisa de argumentos convincentes para persuadirlo de volver (Barchiesi, 1992, p. 91) y mantenerse firme en su postura de no contraer nupcias nuevamente, enfrentando así, la presión paterna. En los versos siguientes, cuenta que su padre desiste de insistir en el asunto sólo cuando percibe que la pietas y la pudicitia de su hija son más fuertes:

Ille tamen pietate mea precibusque pudicis

frangitur et vires temperat ipse suas (Ov. Ep. 1.85-86).

[“Él, sin embargo, se quiebra ante mi piedad y mis púdicas preces y amaina él mismo sus fuerzas.”]

Se ve aquí cómo Penélope e Hipermestra están fuertemente emparentadas por el respeto al matrimonio y por contrariar el mandato paterno. Hipermestra no asesinó a su marido y Penélope decidió urdir un plan para engañar tanto a su padre como a sus pretendientes tejiendo y destejiendo el manto (Ep. 1.19-10), postergando la posibilidad de un nuevo matrimonio. La tenacidad en la defensa de la pietas debida al marido manifestada por estas dos heroínas acaba por legitimarlas como esposas e ideales de mujer.

Las dos mujeres finalmente triunfan en su cometido. Por un lado, el lector sabe que Hipermestra fue liberada del castigo inicialmente impuesto, al tiempo que sus hermanas fueron condenadas precisamente por su impietas, como lo atestiguan tanto Horacio, en la Oda 3.11.30-2 y, posteriormente, también Ovidio, en Metamorfosis 4.462-463. Inclusive, Hipermestra fundará,

${ }^{15}$ La segunda referencia corresponde a Hipsípila (Ep. 6.137). Ella y Penélope comparten el estar legalmente casadas (Hipsípila, al menos, así lo afirma en su epístola: Ep. 6.19-22, 41-44) y esperan el regreso de su marido guardándoles la fidelidad prometida. 
junto a Linceo, una importante dinastía de reyes en Argos. Por otro lado, Penélope verá regresar a su marido después de veinte años de espera. Juntos continuarán al frente del reino de Ítaca, ambos triunfantes en sus objetivos particulares: él, como guerrero y estratego en la guerra de Troya; ella, en la defesa de su matrimonio y de su virtuosismo.

Cánace y su uso argumentativo de la pietas

Canace (Ep. 11) e Hipermestra son dos puellae encadenadas y encarceladas por los propios padres. Los paralelos entre sus epístolas son varios, ya que las dos escriben desde la prisión, mientras se quejan del peso de las cadenas que atan sus manos. Además, escriben a los amados, teniendo, sin embargo, a sus padres como destinatarios ocultos. Con todo, en cada epístola hay una clara diferencia de objetivos: Hipermestra, por un lado, destina de forma transversal la carta al padre para, mediante este recurso, recuperar la libertad. Por otro lado, Cánace sabe que ella debe morir. Su objetivo es salvar la vida del hijo que tuvo con su hermano Macareo, y que fue condenado por el abuelo a ser arrojado a las fieras. Su carta a Macareo es, en verdad, una nota de suicidio, que busca conmover al padre. Este objetivo se encuentra delineado al comienzo de la epístola, en donde la hija de Eolo advierte que su carta podrá estar manchada con su sangre (vv. 1-2). Luego, describe su scribentis imago, ofreciendo la referencia más explícita al acto de escritura en el corpus de las Heroidas (Reeson, 2001, p. 41):

Dextra tenet calamum, strictum tenet altera ferrum,

Et iacet in gremio charta soluta meo.

Haec est Aeolidos fratri scribentis imago;

Sic videor duro posse placere patri. (Ov. Ep. 11.3-6)

["Mi diestra sostiene el cálamo, la otra sostiene la espada desenvainada, y yace en mi regazo el papiro abierto. Esta es la imagen de la hija de Eolo escribiendo a su hermano; así me parece que puedo agradarle a mi duro padre."]

Cánace admite que su embarazo es deshonroso, por eso se refiere a él siempre como crimen. En la Ep. 11, la palabra crimen aparece cuatro veces, siempre en relación al hijo concebido: 
Nec tenui vocem. "Quid”, ait, "tua crimina prodis?” (Ov. Ep. 11.49).

[“Y no contuve la voz. ‘¿Por qué', dijo [la anciana], 'delatas tu crimen?'.’]

Et grave, si morerer, mors quoque crimen erat (Ov. Ep. 11.56).

[“Y, si muriera, la muerte también sería un grave crimen.”]

Et positum est uteri crimen onusque mei (Ov. Ep. 11.64).

["Y fue depositado el crimen y el peso de mi vientre.”]

Crimina sunt oculis subripienda patris (Ov. Ep. 11.66).

[“Debe ocultarse el crimen de los ojos del padre.”]

En otras epístolas del corpus (Ov. Ep. 2.28, 4.31), crimen es utilizado para aludir a la relación entre los amantes. Pero ninguna de las menciones en Ep. 11 se refiere a la relación entre Cánace y Macareo. Esto es un argumento para entender que el error de la puella, tanto para ella como para su padre, no es haber cometido incesto, sino haber concebido un hijo fuera del matrimonio legítimo. ${ }^{16}$ Ella no dice nada respecto a su relación incestuosa.

Los críticos toman diferentes posiciones sobre lo que piensa Eolo con respecto a sus hijos, ya que en la epístola no se encuentra una clara referencia a la postura del padre sobre la relación entre ellos. Tanto Verducci (1985, p. 219220) como Spoth (1992, p. 108-111) opinan que, en la epístola, Cánace acepta el castigo por su incesto (incluso cuando ella no hable expresamente sobre eso), interpretándolo como el leitmotiv de la historia. Verducci (1985, p. 219), por ejemplo, afirma que la existencia del hijo no es tan importante como el hecho de haber nacido del incesto. No hay, sin embargo, indicios textuales (y la autora tampoco los ofrece) que apoyen su lectura.

Casali (1998, p. 707) analiza un irónico verso de la epístola, en que Cánace se queja: quid iuvat... inter cognatos posse referre Iovem? [v. 17, "en que me beneficia poder nombrar a Júpiter entre mis parientes?]. Según el crítico,

no hubiese sido completamente inútil para Cánace apelar a Júpiter, y hubiera sido aun de más ayuda poder apelar a Júpiter como su propio antepasado. Cuando ella pregunta

${ }^{16}$ Da mesma forma, Philippides (1996). 
quid iuvat... inter cognatos posse referre Iovem? la heroína abandona una excelente oportunidad para usar un fuerte argumento a su favor. Entre sus antepasados, Cánace tuvo un dios que sin problemas se casó con su hermana, teniendo de forma subsecuente una gran cantidad de ilustres hijos, sin ningún sentido de culpa (Casali, 1998, p. 707).17

En efecto, la ausencia de tal evidencia en el discurso de Cánace parece una falla en el argumento persuasivo. Sin embargo, es lícito pensar que Ovidio coloca ese verso allí para resaltar la ironía de su situación, pero no hace que Cánace lo use como argumento para defender su relación con Macareo justamente porque el objetivo de su epístola no es sino salvar a su hijo. Además, estrictamente, su situación no es igual a la de Júpiter. Hera y él están casados y el matrimonio es aceptado como tal. Casali, en el pasaje citado, resalta que Júpiter se casó con su hermana y tuvo hijos. No obstante, el problema de Cánace es otro, puesto que en su historia la cronología de los hechos es inversa. Ella tiene un hijo sin estar casada y eso, tal como ella resalta, es crimen.

Bolton compara a Cánace, por su condición de reclusa, no con Hipermestra, sino con Penélope y observa que las dos permanecen en el espacio interior de sus casas como muestra de castidad:

la posición de Cánace dentro de la recámara revela su relación sexual con su hermano y su lealtad hacia él. Su inhabilidad para imaginarse una vida fuera la caracteriza sexualmente como una amante casta (Bolton, 2009, p. 277).18

Sin embargo, es contradictorio que "una amante casta", como Bolton la llama, sienta vergüenza y admita la falta de pudor. La reclusión de Cánace (involuntaria, pero aceptada como castigo por su crimen) es signo de la obediencia paterna y no de la voluntad de preservar su relación amorosa con Macareo. En esto, se aleja de Hipermestra, que sufre su reclusión y pretende

\footnotetext{
17 "it would not have been at all useless for Canace to appeal to Jupiter, and it would have been of even more help for her to be able to appeal to Jupiter as her own forefather. When she asks quid iuvat... inter cognatos posse referre Iovem? the heroine gives up an excellent opportunity to use a strong argument in her favour. Among her forefathers, Canace had a god who had calmly married his own sister, subsequently having a large number of illustrious children, without any sense of shame"

18 "Canace's position within the bedchamber reveals her sexual relationship with her brother and her loyalty towards him. Her inability to envision a life outside characterizes her sexually as the chaste lover."
} 
siempre ganar el perdón paterno para liberarse de esa situación, y de Penélope, que contraría a su padre y no cede ante la exigencia de volver a casarse.

Para remediar su falta de pudicitia y castitas, Cánace defiende su relación con Macareo por ser ésta fruto del amor; un amor desconocido, de acuerdo con el modelo elegíaco, que le causa un padecimiento incontrolable (vv. 25-32). Además, identificándose con el modelo de puella elegíaca característico de las Heroidas, ella describe su pasión a partir de la inocencia de una muchacha que está descubriendo un sentimiento al que el pudor se opone (v. 35). Cánace hace una lista de una serie de síntomas del padecimiento, lugar común en la poesía amatoria. Así, la trama presentada por Ovidio se diferencia de la conocida por medio de la tragedia Eolo, de Eurípides, considerada su principal fuente. En la tragedia, enamorado de ella, Macareo viola a la hermana.

Fulkerson, en su estimulante estudio sobre las Heroidas publicado en 2005, establece relaciones entre las epístolas, y analiza la influencia sincrónica que tienen una(s) sobre la otra(s). Las semejanzas entre las epístolas 11 y 14 la llevaron a afirmar que Hipermestra habría utilizado la epístola de Cánace como modelo intratextual para redactar la propia carta de modo correcto para alcanzar su cometido (Fulkerson, 2005, p. 67-86). Según la autora, Cánace, después de escribir su carta, se suicida con la espada dejada por su padre. Hipermestra, por su parte, redacta la carta para Linceo apropiándose de las estrategias retóricas de Cánace, tras haber aprendido de sus errores. De esta forma, Hipermestra cumple su objetivo y no es obligada a suicidarse. Además de esto, siempre según la autora, Cánace fracasa en persuadir a su padre para que le perdone la vida, pero tiene éxito en alcanzar su verdadero deseo que es salvar la vida de su hijo (Fulkerson, 2005, p. 83).

Sin embargo, analizando el texto, no encontramos referencias textuales a un pedido de ayuda de Cánace a su padre o a Macareo para escapar del suicidio que estaba decidida a cometer. Hipermestra, por el contrario, sí le escribe a Linceo expresamente para que él la rescate del castigo paterno, alegando inocencia. Por lo tanto, cada una tiene un objetivo diferente. Cánace no falla en persuadir para salvarse, ya que ese ni siquiera es su objetivo.

Fulkerson no justifica por qué no entiende la influencia de una puella sobre la otra en la dirección contraria, como si fuera Cánace quien interpreta la epístola de Hipermestra como modelo. Desde nuestro punto de vista, este tipo 
de influencia tendría más sentido, ya que Hipermestra, como fue demostrado supra, es paradigmática por su pietas. Su carácter se aproxima al de Penélope, también ejemplar, por lo que la epístola de Hipermestra sería más adecuada para servir como modelo que la de una puella que decide morir para expiar sus faltas.

Como ya fue dicho, la pérdida de la castitas y de la pudicitia aleja a Cánace del modelo de mujer establecido en el comienzo de las Heroidas con la figura de Penélope. Ella debe aplacar la furia del padre. Para eso, apoya su base argumentativa en el mismo recurso que Hipermestra refuerza al final del corpus: la pietas. Pero, a diferencia de la hija de Dánao, Cánace sí buscará honrar la pietas paterna, única cualidad que le resta después de ser culpable de incesto, haber perdido su castitas y pudicitia (inruit et nostrum vulgat clamore pudorem $^{19}$ [v. 79 "irrumpe y divulga mi deshonra con sus gritos"]; ipsa nihil prater lacrimas pudibunda profudi [v. 81 "yo misma, avergonzada, no derramé nada más que lágrimas]) y haber engendrado un hijo fuera del matrimonio. Solo le queda mostrarse piadosa y obedecer el mandato paterno:

\section{'Aeolus hunc ensem mittit tibi' (tradidit ensem)}

'et iubet ex merito scire quid iste velit.'

Scimus et utemur violento fortiter ense (Ov. Ep. 11.95-97).

[“'Eolo te envía esta espada' (él me entrega la espada) 'y ordena que sepas a partir de tu acción lo que él quiere'. Sé y usaré la violenta espada de forma corajosa.”]

No obstante, Cánace no está de acuerdo con su padre, a quien califica como durus (6), ferus (9), truculentior Euris (9) e inimicus (89). Esto es evidencia de que su decisión de obedecerle tendrá que ver únicamente con su observación de la pietas. Además, ella se muestra completamente dispuesta a resignar su propia vida para que su hijo sea perdonado.

\section{Conclusión}

Los rasgos que caracterizan a Penélope como esposa ideal, esto es, su fidelidad y devoción por el marido, descritos en su epístola ubicada en la apertura del corpus de las Heroidas, se repiten en el carácter extremadamente

19 "a source of shame, dishonour, humiliation" (OLD, ad loc.). 
piadoso de Hipermestra para con su marido en el final del corpus. No es sorprendente, pues, que las dos mujeres sean exitosas en sus relaciones amorosas y que, al contrario de la gran mayoría de las heroínas que escriben en este epistolario, ellas puedan reunirse finalmente con sus amados. ${ }^{20}$

Por otro lado, a partir del análisis de la Ep. 11 se concluyó que es posible una lectura in tandem de las epístolas 11 y 14. La ejemplaridad de Hipermestra permite deducir su función como modelo en el discurso retórico-argumentativo de Cánace, que adapta los argumentos a los propios objetivos. Las virtudes paradigmáticas representadas por Penélope e Hipermestra están ausentes en la epístola de Cánace. Sin embargo, hay comportamiento ejemplar en la forma en que resuelve actuar y comportarse para resarcir el daño. En vez de justificar su error, como lo hace, por ejemplo, Fedra (Ep. 4), ella lo reconoce y busca destacar su personalidad virtuosa mediante la obediencia a su padre.

De esta forma, retomando la idea expuesta al comienzo de este trabajo de que las Heroidas pueden ser leídas como un corpus que complementa las enseñanzas dejadas en el Ars amatoria, tenemos aquí, a modo de ilustración, mujeres que presentan a su modo características ejemplares. Penélope e Hipermestra, sobre todo, gozan de mayor prestigio como figuras paradigmáticas de fidelidad marital y eso es resaltado por su posición enfática, abriendo y cerrando el corpus de las Heroidas míticas.

Por último, es importante observar que, de modo elocuente, Hipermestra cierra su epístola expresando un deseo: scribere plura (Ep. 14.131 [“escribir más”]). Este deseo es el último de su carta y, consecuentemente, también del grupo de las primeras catorce que conforman las Heroidas. Por lo tanto, el corpus de epístolas elegíacas escritas por mujeres colocadas en el papel de poetae/amatrices termina con el deseo de seguir escribiendo. De esta forma, plura es un indicio de que Hipermestra, como probablemente otras mujeres, tendrían más para decir que aquello que fue plasmado. En consonancia con lo observado por Reeson (2001, p. 314), la única opción que les queda a los hombres para saber qué más tienen para decir sus mujeres sería, finalmente, volver con ellas.

${ }^{20}$ Además de ellas, Briseida (Ep. 3) y Hermíone (Ep. 8) también lo hacen. Las dos comparten características en común ya que fueron raptadas y escriben al hombre que aman para ser rescatadas. Briseida, como esclava de Aquiles, representa en sí misma el tópos elegíaco del servitium amoris. Hermíone esclava de Pirro, le escribe a Orestes, a quien ella reconoce como su marido, por haber sido prometida a él antes que a Pirro. 


\section{REFERÊNCIAS BIBLIOGRÁFICAS}

\section{Fuentes Primarias}

Horace. Odes and Epodes. Editado y traducido por Niall Rudd. Cambridge: Harvard University Press, 2004.

P. Ovidius Naso. Amores, Epistulae, Medicamina faciei femineae, Ars amatoria, Remedia amoris. R. Ehwald edidit ex Rudolphi Merkelii recognitione. Leipzig: B. G. Teubner, 1907.

Propertius. Elegies. Editado y traducido por G. P. Goold. Cambridge: Harvard University Press, 1990.

\section{Bibliografia Crítica}

Barchiesi, Alessandro. P. Ovidii Nasonis epistulae Heroidum 1-3. Firenze: Le Monnier, 1992.

Bolton, M. Catherine. Gendered spaces in Ovid's Heroides. Classical World: A Quarterly Journal on Antiquity, v. 3, n. 102, 2009, p. 273-290.

Casali, Sergio. Ovid's Canace and Euripides' “Aeolus": two notes on "Heroides” 11, Mnemosyne, v. 51, fasc. 6, 1998, p. 700-710.

Dörrie, Heinrich. Untersuchungen zur Überlieferungsgeschichte von Ovids Epistulae Heroidum. Göttingen: Vandenhoeck \& Ruprecht, 1960.

P. Ovidii Nasonis Epistulae Heroidum (quas ad fidem codicum edidit). Berlin: Walter de Gruyter, 1971.

Farrel. Joseph. Reading and writing the Heroides. HSCP, vol. 98, 1998, p. 307338.

Fulkerson, Laurel. The Ovidian Heroine as Author: Reading, Writing, and Community in the Heroides. Cambridge: Cambridge University Press, 2005.

Holzberg, Niklas. Ovid. Dichter und Werk. München: C. H. Beck, 1997.

Jacobson, Howard. Ovid's Heroides. Princeton: Princeton University Press, 1974.

Kenney, E. J. Ovid: Heroides XVI-XXI. Cambridge: Cambridge University Press, 1996.

Knox, Peter. Ovid's Heroides: Select Epistles. Cambridge: Cambridge University Press, 1996.

The Heroides: elegiac voices. In: Boyd, Barbara. W. (ed.) Brill's Companion to Ovid. Leiden: Brill, 2002, p.117-39.

Luck, Georg. Untersuchungen zur Textgeschichte Ovids. Heidelberg: Carl Winter, 1969.

Michalopoulos, Andreas. Ovid Heroides 16 and 17. Introduction, text and commentary. Cambridge: Cambridge University Press, 2006.

Philippides, Katerina. Canace misunderstood: Ovid's "Heroides" XI. Mnemosyne, v. 49, n. 4, 1996, p. 426-439. 
Reeson, James. Ovid, Heroides 11, 13, and 14. A commentary. Leiden: Brill, 2001.

Spoth, Friedrich. Ovids Heroides als Elegien. München: Zetemata, 1992.

Stroh, Wilfried. Heroides Ovidianae cur epistolas scribant. In: Papponetti, Giuseppe. (ed.) Ovidio Poeta Della Memoria. Atti del Convegno Internazionale di Studi: Sulmona, 19-21 Ottobre 1989. Roma, 1991, p. 201-44.

Ugartemendía, Cecilia M. Ars oratoria e ars amatoria em Heroides XIV. Ronai, v. 2, n. 2, 2014, p. 173-189.

A Exemplaridade do Abandono. Epístola Elegíaca $e$ Intratextualidade nas Heroides de Ovídio. Dissertação de Mestrado, Letras Clássicas, DLCV/FFLCH, Universidade de São Paulo, 2017.

Verducci, Florence. Ovid's Toyshop of the Heart: Epistulae Heroidum. Princeton: Princeton University Press, 1985. 